\title{
Absolute Distance Metrology for Space Interferometers
}

\author{
Bas L. Swinkels ${ }^{* a}$, Nandini Bhattacharya ${ }^{a}$, Arno A. Wielders ${ }^{b}$ and Joseph J.M. Braat ${ }^{a}$ \\ ${ }^{a}$ Optics Research Group, Delft University of Technology, \\ Lorentzweg 1, 2628 CJ, Delft, The Netherlands; \\ ${ }^{b}$ TNO-TPD, Delft, The Netherlands
}

\begin{abstract}
Future space missions, among which the Darwin Space Interferometer, will consist of several free flying satellites. A complex metrology system is required to have all the components fly accurately in formation and have it operate as a single instrument. Our work focuses on a possible implementation of the sub-system that measures the absolute distance between two satellites with high accuracy. For Darwin the required accuracy is on the order of 70 micrometer over a distance of 250 meter.

We are exploring a technique called frequency sweeping interferometry, which involves interferometrically measuring a phase difference while sweeping the wavelength of a tunable laser. This phase difference is directly proportional to the absolute distance. A very high finesse Fabry-Pérot cavity is used as a reference standard, to which the laser is locked end-points of the sweep. We will discuss the control system that drives the setup and show some first experimental results.
\end{abstract}

Keywords: Absolute distance metrology, Fabry-Pérot cavity, laser stabilization

\section{INTRODUCTION}

In the next few years a number of space missions will be carried out that consist of multiple satellites that fly in close formation. Examples include missions for gravitational wave detection, X-ray telescopes and synthetic aperture telescopes. For most of these, a metrology system that controls the formation with high accuracy is an essential component to achieve the science measurement. The background of our research is the Darwin Infrared Space Interferometer, which will be launched by ESA around 2014 and is aimed at detecting planets around other stars. It consists of 6 free-flying telescopes and a central satellite that will interferometrically combine the collected light to form 'white-light' fringes in the infrared.

The interferometric detection poses very high demands on the satellite pointing and the stability of the mutual distances. It is necessary that the optical path length experienced by the starlight is equal along the different paths from telescopes to beam combination to within a fraction of the used wavelengths. This is not possible without a complex metrology system that monitors all the distances, angles and velocities in the system. ${ }^{1}$ The measurements made with the various systems will be used to control the optical path lengths by moving delay lines and by steering the satellites with milli- and micro-Newton thrusters. Our research focuses on the possible implementation of one of these sub-systems that should measure the absolute distance between two satellites with high accuracy. For Darwin the required accuracy would be slightly better than 100 micrometer over a distance of up to 250 meter.

Several techniques are possible for measuring absolute distances. Some of these are incoherent, such as pulsed time-of-flight measurement and some high frequency modulation schemes. Other methods are coherent and use various interferometric methods. Among these are multiple-wavelength interferometry and several schemes involving tunable lasers. We are currently investigating a scheme that uses only one tunable laser, which is known as frequency sweeping interferometry. ${ }^{2,3}$ The technique basically works by sweeping a tunable laser over a well known frequency difference. The total phase difference observed is then directly proportional to the optical path length difference in the interferometer.

\footnotetext{
* e-mail: b.l.swinkels@tnw.tudelft.nl
} 


\section{THEORY}

Consider an optical interferometer with a fixed optical path length difference $L$ that is equipped to measure phase as a function of time. For fixed or slowly changing optical frequency $\nu$, the phase $\phi$ is proportional to both the length and the frequency. Because the phase is usually measured modulo $2 \pi$, the absolute phase is unknown. By unwrapping the phase over time it is however possible to measure phase differences. If the light-source is a tunable laser that is swept from optical frequency $\nu_{1}$ to $\nu_{2}$, the total phase difference will be

$$
\phi_{2}-\phi_{1}=2 \pi \frac{\nu_{2} L}{c}-2 \pi \frac{\nu_{1} L}{c}=2 \pi \frac{\Delta \nu}{c} L=2 \pi \frac{L}{\Lambda},
$$

with $c$ the speed of light, $\Delta \nu$ the frequency difference of the sweep and $\Lambda$ the so called synthetic wavelength defined by

$$
\Lambda=\frac{c}{\Delta \nu} .
$$

Reversing Eq. (1), the length $L$ should be calculated as

$$
L=\frac{\phi_{2}-\phi_{1}}{2 \pi} \Lambda=\frac{\Delta \phi}{2 \pi} \Lambda .
$$

The length is thus directly proportional to the total phase difference $\Delta \phi$, which can be determined by measuring the phase before and after the sweep and counting the number of fringes during the sweep. Error analysis of Eq. (3) yields the error in the length measurement $\delta L$

$$
\begin{aligned}
\delta L & =\left[\left(\Lambda \frac{\delta \Delta \phi}{2 \pi}\right)^{2}+\left(L \frac{\delta \Lambda}{\Lambda}\right)^{2}\right]^{1 / 2} \\
& \approx\left[2\left(\Lambda \frac{\delta \phi}{2 \pi}\right)^{2}+2\left(L \frac{\nu}{\Delta \nu} \frac{\delta \nu}{\nu}\right)^{2}\right]^{1 / 2} .
\end{aligned}
$$

The first term is constant and depends on the error in the phase difference $\delta \Delta \phi$, which is determined by two phase measurements with an error $\delta \phi$ each. The second term scales with the length $L$ and depends on the error in the synthetic wavelength $\delta \Lambda$. As indicated by Eq. (2), the synthetic wavelength is determined by a difference between two large frequencies with error $\delta \nu$ each. This leads to the amplification by a large factor $\nu / \Delta \nu$.

One drawback of using frequency sweeping interferometry is that the technique is very sensitive to target movement during the wavelength sweep. A movement over a distance of one optical wavelength would then be interpreted as a shift over one synthetic wavelength. We can largely compensate for this effect with a simple interpolation scheme. ${ }^{4}$ This scheme only works if the movements are smooth on the time-scale of a measurement, which would be the case in our intended application.

\section{EXPERIMENTAL}

See Fig. 1 for a schematic overview of the experimental set-up. The left part of the figure contains the laser and some components to make it tune very accurately over a known frequency interval. The laser is a Littman-type external cavity laser diode with a wavelength of $633 \mathrm{~nm}$. The optical frequency can be tuned continuously over several tens of $\mathrm{GHz}$ with the aid of a piezo-actuator. The laser also has a current modulation input, which is used for high frequency feedback. The right part of the figure describes the interferometer that measures the actual distance.

\subsection{Fabry-Pérot cavity}

The endpoints of the wavelength sweep are defined by locking the laser to a Fabry-Pérot cavity with the PoundDrever-Hall method. This involves modulating the light with an electro-optic modulator and detecting the light reflected from the cavity. Mixing this signal with the modulation frequency yields an error signal that has a very steep slope at the frequency of a cavity resonance. Given sufficient bandwidth of the feedback loop, it is possible 


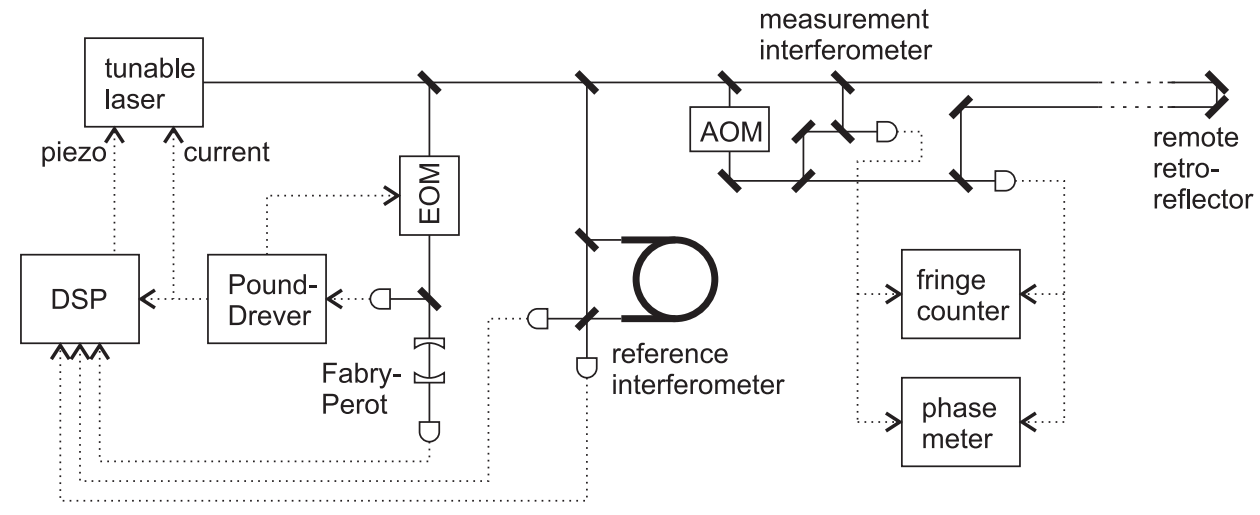

Figure 1. Schematic of the frequency sweeping interferometer, see text for details. AOM and EOM are acousto- and electro-optic modulators.

to narrow the line-width of the laser to well below 100 Hertz. ${ }^{5}$ The cavity is $10 \mathrm{~cm}$ long and is built completely out of ultra-low expansion (ULE) glass. It has a finesse of 10000 and is placed in a small vacuum tank.

The magnitude of the wavelength sweep will be an integer number of free-spectral-ranges of the cavity. The synthetic wavelength of eq. (2) will therefore be directly dependent on the length of the cavity, which thus should be calibrated.

\subsection{Reference interferometer}

To observe and control the optical frequency changes of the laser we built a reference interferometer. It is a homodyne Mach-Zehnder type interferometer with 10 meter fiber in the long branch. A polarization trick is used to obtain two signals in quadrature. ${ }^{6}$ It is used only to measure changes on a short time-scale. Temperature drift of the fiber is not a problem, since the Fabry-Pérot cavity is used for maintaining the long term calibration.

\subsection{Measurement interferometer}

The actual distance measurement is carried out in the measurement interferometer. The phase is measured with a heterodyne scheme, for which the light is modulated with an acousto-optic modulator. The light is interfered twice: once in a short reference path and once in an interferometer that contains the large unknown path length difference. For our intended application this would be formed by a beam-expander and a retro-reflector on the target. To test the system in the lab we will use long fibers instead. To suppress periodic phase non-linearities as much as possible we use separate beams and no polarizing components.

The integer number of fringes during the wavelength sweep will be monitored with a fast counter. For accurately measuring the phase at the endpoints, the heterodyne signals are electronically mixed to a much lower frequency at which the timing of the various zero-crossings is recorded.

\subsection{Control system}

During a measurement the system should take a number of different actions within a few tenths of a second. At one moment the laser should tune very fast over a large frequency span. Subsequently it should slow down and find one of the resonances of the Fabry-Pérot cavity. For some time it should accurately lock to the resonance and a moment later it should sweep back again. This kind of actions are only possible with some form of feedback.

For both the large frequency sweep and the low-frequency locking to the cavity the piezo-input of the laser is used. To achieve the required flexibility a digital signal processor (DSP) that has a number of analog in- and outputs is used. A PID-type controller was implemented which either makes the optical frequency track a predefined waveform or locks the system to the error signal of the Pound-Drever electronics. See Fig. 2 for an overview of the digital feedback system. Not shown in the figure is some logic for searching for a cavity resonance, switching between the two feedback loops and adjusting the waveform to allow for drift in either the laser or the reference 


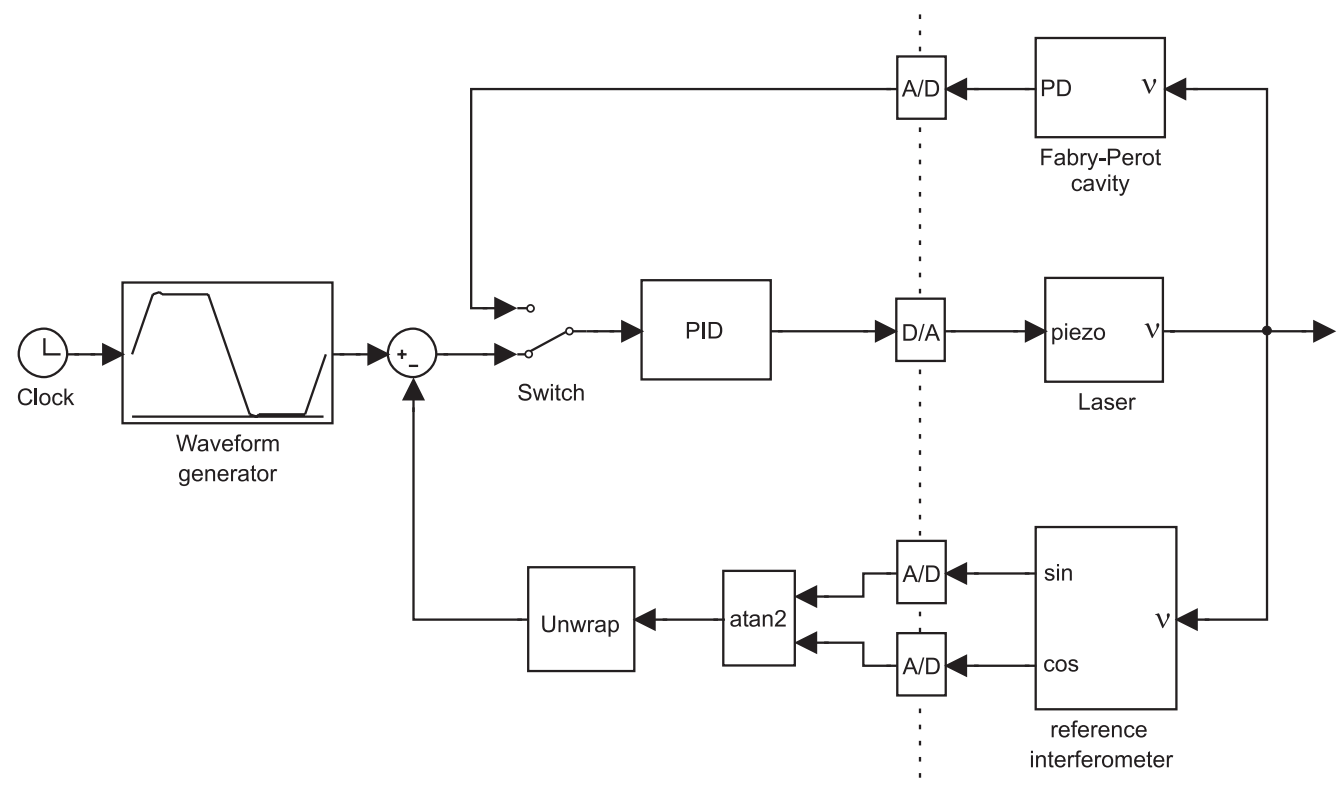

Figure 2. Schematic of the digital feedback. The part to the left of the dotted line is implemented in a digital signal processor, the part on the right consists of optics and electronics. PD is the Pound-Drever error signal, $\nu$ is the optical frequency.

interferometer. This scheme was first simulated with Simulink and later compiled to the DSP. The loop operates at a frequency of $40 \mathrm{kHz}$, well above the bandwidth of the piezo of $2 \mathrm{kHz}$.

The high-frequency part of locking to the cavity is achieved with analog electronics, by feeding a filtered version of the Pound-Drever signal directly to the current of the laser diode. In this way a bandwidth of roughly $1 \mathrm{MHz}$ can be obtained.

\section{RESULTS}

Fig. 3 shows some of the signals that were measured while applying a trapezoidal waveform to the piezo (openloop). At the same time high frequency feedback was applied by connecting the Pound-Drever signal to the laser diode current. By carefully choosing the offset and the amplitude of the input signal the laser is swept exactly over a number of free-spectral ranges of the cavity and it locks to a resonance at both the end-points of the sweep. Fig. 3.a shows the phase, which is obtained by taking the arctangent of the quadrature signals from the reference interferometer. Fig. 3.b shows the transmission of the cavity which shows the locking. Since the line-width of the laser is higher than the line-width of the cavity the transmission does not stay high but shows a noisy pattern. Eventually we hope to narrow the line-width of the laser to below that of the cavity by optimizing the analog feedback loop. Fig. 3.c shows the unwrapped phase of a). This signal closely resembles the input signal, but close inspection shows some non-linear behavior of the piezo, such as hysteresis and creep. The system only operates as shown for some tens of seconds before the laser slowly drifts out of the lock.

Fig. 4 shows the same signals as before, but in this case the system operates totally under control of the digital signal processor. A control scheme similar to Fig. 2 is used and no signal is fed back to the laser diode current. The large sweep no longer shows the non-linear behavior of the piezo but now follows a straight curve. At the end of the sweep the system slows down and starts searching for a resonance. Once a resonance is found the feedback is switched to the Pound-Drever signal for the duration of the lock period. By constantly searching in a small range around the previously found frequency the system is tolerant to some drift in both the laser and the reference interferometer. The locking behavior should further be improved by adding the high frequency current feedback. 


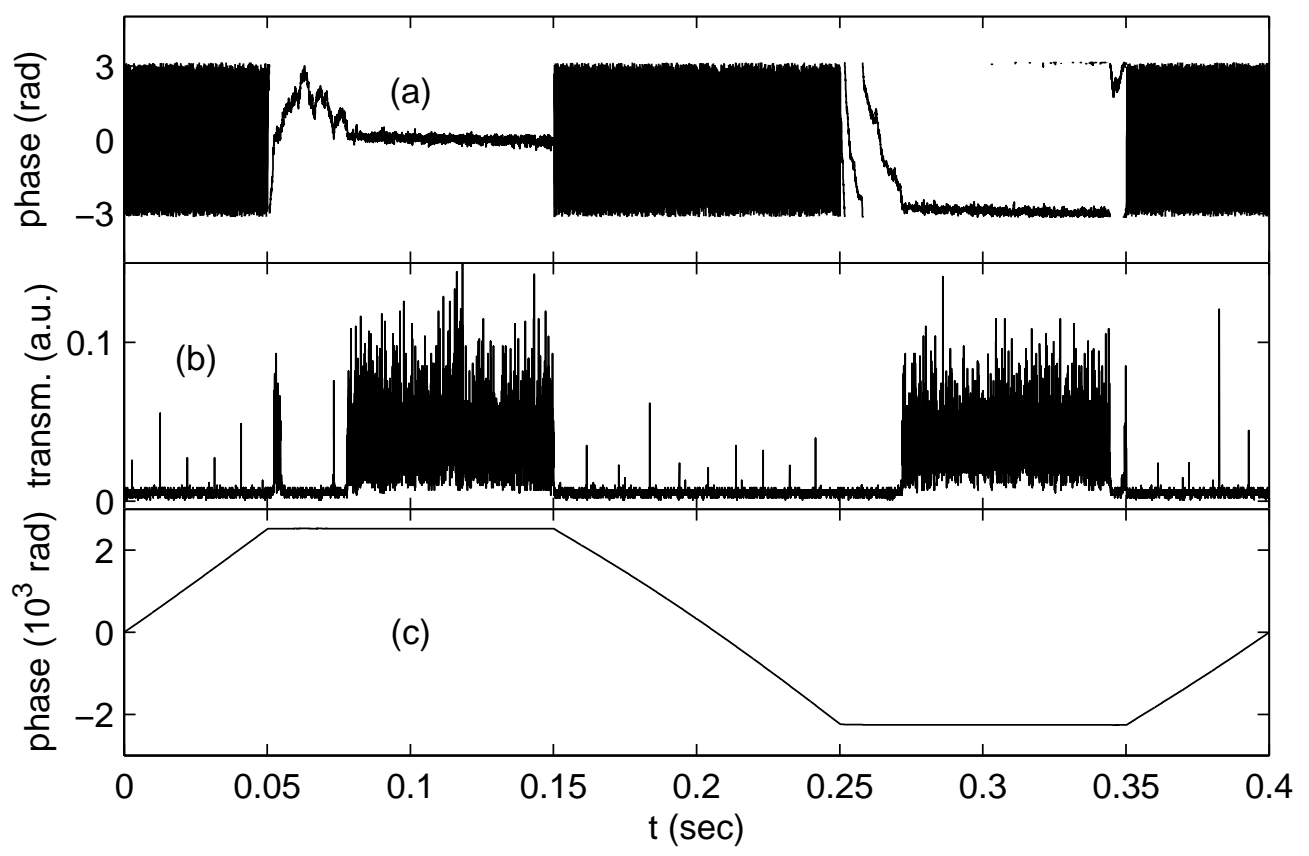

Figure 3. Measurement data. The piezo is scanned open loop and the Pound-Drever signal is fed to the laser diode current. The laser is scanned over 10 free-spectral-ranges of the cavity. a) Interferometric phase. b) Transmission of the Fabry-Pérot cavity. c) Unwrapped phase of a).

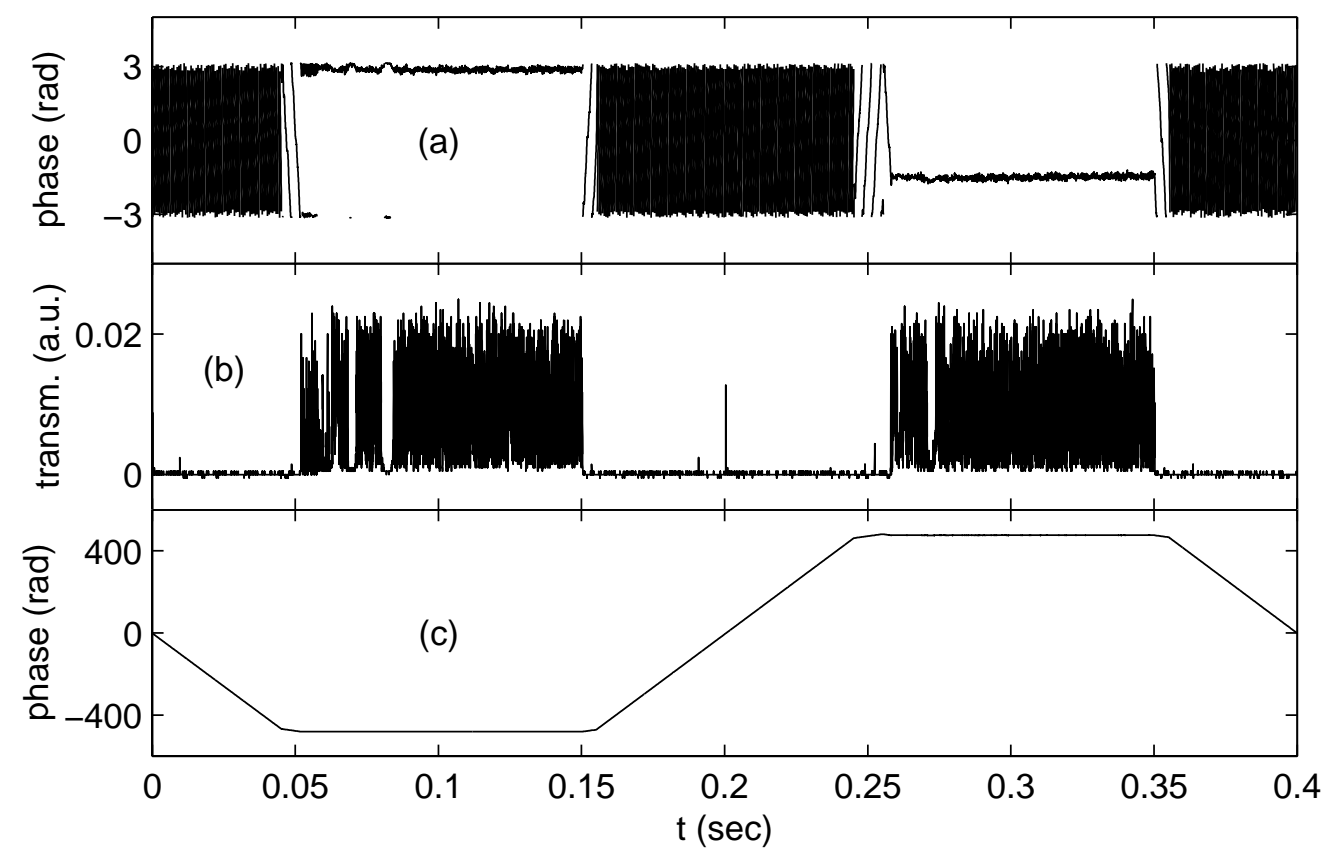

Figure 4. Measurement data. The piezo is swept under control of the digital signal processor, but there is no feedback applied to the laser diode current. The laser is scanned over 2 free-spectral-ranges of the cavity. a) Interferometric phase. b) Transmission of the Fabry-Pérot cavity. c) Unwrapped phase of a). 
The experiments described above also allow for calculating the length of the reference interferometer by evaluating the phase difference of the sweep. The best results obtained so far show a repeatability of 30 micrometer at a distance of 15 meter while scanning the laser over 10 free-spectral-ranges of the cavity. The sensitivity of the system to target movement was also tested by heating the fiber during a measurement run. Due to our correction algorithm a repeatability of 100 micrometer was achieved in the presence of movement, where no correction would have rendered the measurement useless. ${ }^{4}$

\section{CONCLUSIONS}

We described a system for measuring absolute distances which we are currently building. An essential part of our measurement method consists of locking a laser to a high finesse Fabry-Pérot cavity. We succeeded in doing this both with analog electronics and with a digital signal processor, but further tuning of the feedback loops needs to be done.

We also performed some preliminary length measurements, which show a repeatability of 30 micrometer over 15 meter. Furthermore we tested the sensitivity of our system to target movement. Further tests and measurements with longer lengths of the measurement interferometer will be performed in future.

\section{ACKNOWLEDGMENTS}

This project was funded by TNO-TPD, the Applied Physics Division of the Netherlands Organization for Applied

Scientific Research. The research was carried out in the framework of the Knowledge Center Optical Aperture Synthesis, a joint initiative of Delft University of Technology and TNO.

\section{REFERENCES}

1. B. Calvel, I. Cabeza, A. Cabral, E. Manske, J. Rebordao, R. Sesselmann, Z. Sodnik, A. Verlaan, "High Precision Optical Metrology for Darwin: Design and Performance," 6th International Conference on Space Optics, Toulouse, 2004.

2. J. Thiel, T. Pheifer, and M. Hartmann, "Interferometric measurement of absolute distances of up to $40 \mathrm{~m}$," Measurement 16, 1-6 (1995).

3. D. Xiaoli and S. Katuo, "High-accuracy absolute distance measurement by means of wavelength scanning heterodyne interferometry", Meas. Sci. Technol. 9, 1031-1035 (1998).

4. B.L. Swinkels, N. Bhattacharya, and J.J.M. Braat, "Correcting movement errors in frequency sweeping interferometry", submitted to Optics Letters.

5. A. Schoof, J. Grünert, S. Ritter and A. Hemmerich, "Reducing the linewidth of a diode laser below $30 \mathrm{~Hz}$ by stabilization to a reference cavity with a finesse above 10" , Optics Letters 26, 1562-1564 (2001).

6. Th. Kinder and K.-D. Salewski, "Absolute distance interferometer with grating-stabilized tunable diode laser at $633 \mathrm{~nm}, "$ J. Opt. A 4, S364-S368 (2002). 